\title{
LARGE-SCALE EXPANSION AND CHARACTERIZATION OF HUMAN ADULT NEURAL CREST-DERIVED MULTIPOTENT STEM CELLS FROM HAIR FOLLICLE FOR REGENERATIVE MEDICINE APPLICATIONS
}

\author{
R.G. Vasyliev ${ }^{1,2, *}$, A.E. Rodnichenko ${ }^{1,2}$, O.S. Gubar ${ }^{2,3}$, A.V. Zlatska ${ }^{1,2}$, I.M. Gordiienko ${ }^{2,4}$, \\ S.N. Novikova ${ }^{1}$, D.O. Zubov ${ }^{1,2}$ \\ ${ }^{1}$ State Institute of Genetic and Regenerative Medicine, National Academy of Medical Sciences of Ukraine, \\ Kyiv 04114, Ukraine \\ ${ }^{2}$ Biotechnology Laboratory ilaya.regeneration, Medical Company ilaya ${ }^{\circledR}$, Kyiv 03115, Ukraine \\ ${ }^{3}$ Institute of Molecular Biology and Genetics, NAS of Ukraine, Kyiv 03680, Ukraine \\ ${ }^{4}$ R.E. Kavetsky Institute of Experimental Pathology, Oncology and Radiobiology, NAS of Ukraine, \\ Kyiv 03022, Ukraine
}

Aim: The purpose of this work was to obtain, multiply and characterize the adult neural crest-derived multipotent stem cells from human hair follicle for their further clinical use. Materials and Methods: Adult neural crest-derived multipotent stem cells were obtained from human hair follicle by explant method and were expanded at large-scale up to a clinically significant number. The resulted cell cultures were examined by flow cytometry and immunocytochemical analysis. Their clonogenic potential, ability to self-renewal and directed multilineage differentiation were also investigated. Results: Cell cultures were obtained from explants of adult human hair follicles. Resulted cells according to morphological, phenotypic and functional criteria satisfied the definition

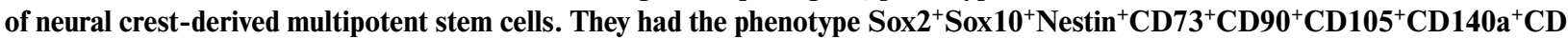

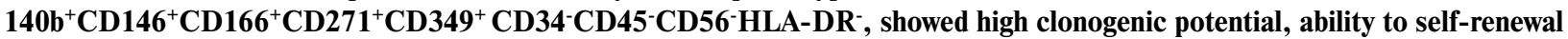
and directed differentiation into the main derivatives of the neural crest: neurons, Schwann cells, adipocytes and osteoblasts. Conclusion: The possibility of a large-scale expansion of adult neural crest-derived multipotent stem cells up to 40-200-10 from minimal number of hair follicles with retention of their phenotype and functional properties are the significant step towards their translation into the clinical practice.

Key Words: regenerative medicine, neural crest, hair follicle, neural crest-derived multipotent stem cells, directed differentiation, large-scale expansion.

Regenerative medicine is a rapidly developing field that promises to significantly improve the quality of the clinical treatment and to develop the cure for incurable diseases and pathological conditions. The main tools of regenerative medicine are tissue engineering, cell therapy and gene therapy. Nowadays there are several tissue-engineering, cellular and gene medical products in the world, successfully passed clinical trials and emerged on the market [1]. Most methods of regenerative medicine are based on in vitro culturing of human stem, progenitor or differentiated cells

Submitted: August 08, 2017.

${ }^{\star}$ Correspondence: $\quad$ E-mail: rvasyliev.ilaya@gmail.com Tel.: +380992750434

Abbreviations used: BDNF - brain-derived neurotrophic factor; bFGF - basic fibroblast growth factor; BSA - bovine serum albumin; CFU - colony forming units; EGF - epidermal growth factor; ELISA - enzyme-linked immunosorbent assay; FBS - fetal bovine serum; GDNF - glial cell line-derived neurotrophic factor; GMP/ GTP - good manufacturing practice/good tissue practice; HF - hair follicle; IGF - insulin-like growth factor; ITS - insulin-transferrinselenite supplement; LNGFR - low-affinity nerve growth factor receptor; MSCs - multipotent mesenchymal stem/stromal cells; NCMSCs - neural crest-derived multipotent stem cells; NGF - nerve growth factor; $\mathrm{P}$ - passage; $\mathrm{PCR}$ - polymerase chain reaction; PDGF - platelet-derived growth factor; PDN - population doubling number; PDT - population doubling time; PE - plating efficiency; $\mathrm{SCl}$ - spinal cord injury; SKPs - skin-derived precursors cells. to increase their number up to required for clinical use, as well for the genetic correction, modifications of their biological properties or to create $3 \mathrm{D}$ living equivalents of different tissues and organs. Despite the significant progress in the field of pluripotent stem cells (embryonic stem cells and induced pluripotent stem cells), a number of ethical and biosafety issues remain unresolved. Thus, the identification and characterization of new types of adult stem cells is an actual challenge. In addition, the medical use of in vitro cultured human cells requires the development of criteria for the safety and efficacy of cell-based medicinal products in terms of identity, purity and potency. For example, such criteria were developed for multipotent mesenchymal stem/stromal cells (MSCs) from bone marrow and adipose tissue [2, 3].

Adult neural crest-derived multipotent stem cells (NC-MSCs) for a number of reasons are a promising cell type for regenerative medicine applications [4]. During the embryonic development, neural crest cells generate a wide variety of cell types and tissues, such as: bone, cartilage and connective tissue of the head and neck; melanocytes; endocrine cells (thyroid gland C-cells, adrenal medulla); neurons and glia of the peripheral nervous system and others. Adult NC-MSCs are identified and isolated from many tissues and organs of the adult mammalian organism, including 
humans [5]. It is shown that adult NC-MSCs retain the ability to directed multilineage differentiation into the main derivatives of the neural crest, similar to their embryonic counterparts [5]. In a number of studies on animal models the positive effect of adult NC-MSCs has been shown to restore the critical size of calvarial bone defects [6], stimulation of peripheral nerve regeneration [7, 8], spinal nerve dorsal root avulsion [9] and spinal cord injury treatment [10].

One of the most attractive from the possible sources of adult NC-MSCs is the hair follicle (HF) due to the minimal invasiveness of the biopsy. For the first time, adult NC-MSCs were identified and isolated from mouse whisker follicles [11]. The method was based on the use of the explant technique and a migratory behavior of NC-MSCs. Later, adult NC-MSCs were obtained from skin HFs of mice and another species, including human [12-14]. For the production of human adult NC-MSCs, several techniques have been proposed: the culturing of HF explants plucked [15] or dissected from a skin biopsy [16], selection of cells from dissociated HFs based on surface markers by fluorescence activated cell sorting or immunomagnetic separation [17], culturing dissociated cells under sphere-forming conditions, etc [18]. Each of these techniques has its advantages and disadvantages. However, even when using similar techniques for the production of human adult NC-MSCs, the resulting populations differ in the expression of a number of key neural crest markers such as Sox 10 and p75 (CD271, LNGFR) [15, 16]. Also, minimal data are available on the expression of surface markers by cultured human adult NC-MSCs and on the possibility of their large-scale expansion to obtain the significant cell number required for clinical applications.

Thus, the goal of our work was to obtain human adult NC-MSCs cultures from the minimal skin biopsy of patients, to master their large-scale expansion, and to determine their phenotype and functional properties.

\section{MATERIALS AND METHODS}

All procedures for obtaining human skin biopsies, cell isolation and culturing were performed with written informed consent of patients and in accordance with the laws of Ukraine. The study protocol and the local clinical protocol were approved by the Bioethics Committee of the Medical company ilay ${ }^{\circledR}$. Cell culturing was carried out in the GMP/GTP-compliant biotechnological laboratory ilaya.regeneration ( $\mathrm{Li}$ cense to operate the Banks of human cord blood, other tissues and cells, issued by the Ministry of Health of Ukraine AE No. 186342 from 11.07.2013).

The donor skin specimens were obtained from 3 patients with spinal cord contusion injuries and 2 patients with critical sized calvarial bone defects.

Donors and cells screening. All patients/ donors (peripheral blood - ELISA, PCR) and cell cultures (PCR) were screened for absence of human immunodeficiency virus type $1 / 2$, hepatitis $B$ virus, hepatitis $C$ virus, herpes simplex virus type $1 / 2$, cytomegalovirus, Epstein - Barr virus, Treponema pallidum and Mycoplasma ssp. The cell cultures were also tested for common mycoplasma contamination by PCR with Mycoplasma Test Kit I (AppliChem, Germany). The normal karyotype of cultured cells was confirmed by GTG-banding method.

The isolation and culturing of NC-MSCs from human HF. The NC-MSCs cultures were obtained from HF explants according to M. Sieber-Blum method [11] in our modification. One skin specimen per donor was obtained under local anesthesia with use of a dermal punch (diameter $5 \mathrm{~mm}$ ). The biopsies were placed in DMEM:F12 transport medium (Sigma, USA) supplemented with $15 \mathrm{mM}$ HEPES and antibiotic/antimycotic solution. Skin specimen was minced and incubated overnight at $4^{\circ} \mathrm{C}$ in Dispase II solution $(0.4 \mathrm{U} / \mathrm{ml})$ (Sigma-Aldrich, USA). The next day, under the control of Stemi 2000 stereomicroscope (Carl Zeiss, Germany), a layer of epidermis was removed using tweezers, and then HFs were isolated. HFs were explanted over a thin layer of collagen gel in two $35 \mathrm{~mm}$ Petri dishes and incubated for $\mathbf{4 0} \mathrm{min}$. After attachment, the explants were covered with a growth medium and cultured in a multi-gas incubator Binder CB 210 (Binder, Germany) at $37^{\circ} \mathrm{C}$ with $5 \% \mathrm{CO}_{2}$ and $5 \% \mathrm{O}_{2}$ and a saturating humidity. For expansion of adult NC-MSCs, the following growth medium was used: aMEM w/o nucleosides (Sigma-Aldrich, USA), 5\% FBS (Sigma-Aldrich, USA), $5 \mathrm{ng} / \mathrm{ml} \mathrm{bFGF}$ (Gibco, UK), 10 ng/ml EGF (Gibco, UK), 1\% ITS supplement (Gibco, UK), $2 \mathrm{mM}$ stable L-glutamine (BioWest, France) and 1\% antibiotic/antimycotic solution (BioWest, France). The primary culture was passaged on Day 14-20 into the collagen-coated T25 flasks (SPL, Korea). Starting at P2, the cells were seeded into the collagen-coated $875 \mathrm{~cm}^{2}$ surface area multi-flasks (Corning, USA) with a seeding density of 1000 cells $/ \mathrm{cm}^{2}$. The cultures were subcultured using $0.01 \%$ trypsin solution in $0.53 \mathrm{mM} \mathrm{Na}_{2}$ EDTA solution (Sigma-Aldrich, USA). The cell population doubling number (PDN) and cell population doubling time (PDT) were calculated according to the following standard formulas [19]:

$$
\begin{gathered}
P D T=T / 3.31 \lg \left(X_{k} / X_{0}\right) ; \\
P D N=3.31 \lg \left(X_{k} / X_{0}\right),
\end{gathered}
$$

where $X_{k}-$ number of obtained cells; $X_{0}$ - number of plated cells; $T$ - cell culture time.

Colony forming units (CFU) assay and self-renewal ability. To assess the clonogenic potential, the NC-MSCs at the last passage $(P)$ were seeded in number of 100-300 cells in collagen-coated $100 \mathrm{~mm}$ Petri dishes (SPL, Korea) in growth medium supplemented with $20 \%$ FBS and cultured for 14 days. The cells were either fixed and stained for CFU assay or subcloned for self-renewal analysis. The effectiveness of colony formation (plating efficiency) was calculated using the standard formula [19]:

$$
\begin{aligned}
P E, \%= & (\text { no. colonies counted } / \text { no. cells } \\
& \text { inoculated }) \times 100,
\end{aligned}
$$

where $\mathrm{PE}-$ plating effciency. 
Transfer of the clonal colony (subcloning) was carried out using cloning cylinders (Sigma-Aldrich, USA). The cells were cultured for 14 days, fixed and stained for analysis for formation of secondary CFUs.

Analysis of the expression of cell surface and intracellular markers by flow cytometry. NCMSCs immunophenotype was determined at the final passage using a flow cytometer-sorter BD FACSAria (BD Biosciences, USA) with fluorochrome-conjugated mouse monoclonal antibodies to CD34, CD45, CD56, CD73, CD90, CD105, CD140a, CD140b, CD146, CD166, CD271 and Nestin (BD Pharmingen, USA) in accordance with the manufacturer's instructions. For detection of Sox10, primary unconjugated mouse monoclonal antibodies (Novus, USA) and secondary Alexa-647 donkey anti-mouse monoclonal antibodies (Thermo Fisher, USA) were used. For the detection of CD349 (Frizzled-9), primary unconjugated rabbit antibodies (Bioss, USA) and secondary Alexa-647 donkey anti-rabbit monoclonal antibodies were used. For intracellular staining cells were fixed with Cytofix solution, permeabilized and stained in PhosFlow buffer (all - BD Biosciences, USA) according to the manufacturer's instructions. The assay was performed using BD FACS Diva 6.1 software (BD Biosciences, USA). Histogram generation was performed with use of software Cyflogic v.1.2.1 (CyFlo Ltd., USA).

Directed adipogenic differentiation. NC-MSCs were seeded with density of $40 \cdot 10^{3}$ cells $/ \mathrm{cm}^{2}$ in growth medium and the following day were switched to the following differentiation medium: DMEM-HG $(4.5 \mathrm{~g} / \mathrm{l})$ supplemented with $10 \%$ FBS, $1 \mu \mathrm{M}$ dexamethasone, $200 \mu \mathrm{M}$ indomethacine, $500 \mu \mathrm{M}$ isobutylmethylxanthine, $5 \mu \mathrm{g} / \mathrm{ml}$ insulin (all - Sigma-Aldrich, USA) and $5 \%$ horse serum (BioWest, France). The medium was changed 2 times a week. The duration of differentiation was 14 days.

Directed osteogenic differentiation. NC-MSCs were seeded at $20 \cdot 10^{3}$ cells $/ \mathrm{cm}^{2}$ in growth medium and the next day switched to a following differentiation medium: DMEM low glucose $(1.0 \mathrm{~g} / \mathrm{l})$ with $10 \%$ FBS, $100 \mathrm{nM}$ dexamethasone, $10 \mathrm{mM} \beta$-glycerophosphate and $50 \mu \mathrm{g} / \mathrm{ml}$ ascorbate-2-phosphate (all - SigmaAldrich, USA). The medium was changed 2 times a week during 21 days.

Directed neuronal differentiation. NC-MSCs were seeded at a concentration of $50 \cdot 10^{3}$ cells on coated by poly-L-lysine (Sigma-Aldrich, USA) and laminin (Gibco, UK) coverslips in 4 well plates (SPL, Korea) in growth medium. The following day cells were switched to the following differentiation medium: Neurobasal medium (Gibco, UK), 2\% B27 supplement (Gibco, UK), 1\% N2 supplement (Gibco, UK), $5 \mu \mathrm{M}$ ec32 synthetic retinoid (AMSBIO, UK), $1 \mu \mathrm{M}$ forskolin (Sigma-Aldrich, USA), $20 \mathrm{ng} / \mathrm{ml}$ NGF (PeproTech, USA), $20 \mathrm{ng} / \mathrm{ml}$ BDNF (PeproTech, USA), 20 ng/ml GDNF (PeproTech, USA) and $20 \mathrm{ng} / \mathrm{ml}$ IGF (Gibco, UK). The duration of differentiation was 14 days.
Differentiation in the glial direction (Schwann cells). NC-MSCs were seeded at a concentration of $50 \cdot 10^{3}$ cells on coated by poly-L-lysine (SigmaAldrich, USA) and laminin (Gibco, UK) glass coverslips in 4 well plates (SPL, Korea) in growth medium. The following day cells were switched to the following differentiation medium: 1:1 of Neurobasal (Gibco, UK) and DMEM:F12 (Gibco, UK) media, 2\% B27 supplement (Gibco, UK), 1\% N2 supplement (Gibco, UK), $1 \mu \mathrm{M}$ ec32 synthetic retinoid (AMSBIO, UK), $5 \mu \mathrm{M}$ forskolin (Sigma-Aldrich, USA), $20 \mathrm{ng} / \mathrm{ml}$ Neuregulin-1 (Gibco, UK), 10 ng/mI PDGF-BB (PeproTech, USA) and $20 \mathrm{ng} / \mathrm{ml}$ IGF (Gibco, UK). The duration of differentiation was 14 days.

Cytochemistry. For CFU staining, the cell colonies were fixed for 20 min with cold ethanol and azure-eosin stained by Romanowsky (Makrokhem, Ukraine) for $20 \mathrm{~min}$. To confirm the osteogenic and adipogenic differentiation, the cells were fixed for $20 \mathrm{~min}$ in $10 \%$ formalin (Makrokhem, Ukraine), washed with phosphate buffered saline (Sigma-Aldrich, USA) and stained for 20 min with $2 \%$ solution of Alizarin Red S ( $p H ~ 4.1$; for detecting calcified extracellular matrix) and $0.5 \%$ solution of Oil Red $\mathrm{O}$ (for staining of neutral lipids) respectively (all - Sigma-Aldrich, USA).

Immunocytochemistry. The following primary antibodies were used for immunocytochemical staining: rabbit polyclonal against $\mathrm{S} 100 \beta$ (Invitrogen, USA), p75 (CD271) and Sox2 (all — BioLegend, USA); mouse monoclonal against Sox10 (R\&D, USA), Nestin (Santa Cruz, USA), $\beta$-III-tubulin (Sigma-Aldrich, USA). Secondary antibodies were donkey anti-mouse and donkey anti-rabbit Alexa-488 or Alexa-647 conjugated (Thermo Fisher, USA). The cells were fixed for 20 min with cold $4 \%$ paraformaldehyde, permeabilized with intracellular staining for 15 min with $0.1 \%$ Triton X-100, blocked for 30 min in phosphate buffered saline with $0.1 \%$ Tween-20, 1\% BSA, 5\% FBS. The slides were incubated with primary antibodies overnight at $40{ }^{\circ} \mathrm{C}$ and with secondary antibodies for 1 hour at room temperature.

Microscopy. Intravital microscopy and examination of cytological slides were carried out with inverted Axio Observer A1 microscope equipped with an AxioCam ERc 5s digital camera and ZEN 2012 software. Confocal microscopy was performed with Zeiss LSM 510 META microscope (all - Carl Zeiss, Germany).

Statistics. The data are presented as Mean and Standard Deviation $(\mathrm{M} \pm \mathrm{SD})$.

\section{RESULTS AND DISCUSSION}

We successfully obtained and expanded up to the therapeutic dose the adult NC-MSCs from all 5 donors. The characteristics of donors, number of explanted $\mathrm{HF}$ and terms of growth of primary cell cultures are presented in Table 1. On average, 15.8 $\pm 4.5 \mathrm{HFs}$ were obtained from one skin sample of $5 \mathrm{~mm}$ in diameter. It should be noted that part of HFs (1-5 per culture, $16.4 \pm 7.1 \%$ of explanted HFs) was detached, despite 


\begin{tabular}{|c|c|c|c|c|c|c|c|c|}
\hline $\begin{array}{c}\text { Donor } \\
\text { ID }\end{array}$ & Age, sex and condition & $\begin{array}{c}\text { Total number } \\
\text { of explanted HFs }\end{array}$ & $\begin{array}{l}\text { HFs with NC- } \\
\text { MSCs out- } \\
\text { growth, n (\%) }\end{array}$ & $\begin{array}{l}\text { HFs with mixed } \\
\text { outgrowth, } n(\%)\end{array}$ & $\begin{array}{l}\text { HFs with kera- } \\
\text { tinocytes out- } \\
\text { growth, n (\%) }\end{array}$ & $\begin{array}{l}\text { Detached } \\
\text { HFs, n (\%) }\end{array}$ & $\begin{array}{c}\text { Culture } \\
\text { time, days }\end{array}$ & Cell yield \\
\hline \multirow{4}{*}{$\begin{array}{l}1 \\
2 \\
3 \\
4\end{array}$} & \multirow{4}{*}{$\begin{array}{l}24 \text { years, male, } \mathrm{SCl} \\
25 \text { years, male, } \mathrm{SCl} \\
31 \text { years, male, } \mathrm{SCl} \\
27 \text { years, male, bone } \\
\text { defect }\end{array}$} & 14 & $3(21.4)$ & $7(23.8)$ & $2(23.8)$ & $1(23.8)$ & 14 & 29,000 \\
\hline & & 9 & $2(33.3)$ & $3(23.8)$ & $3(23.8)$ & $1(23.8)$ & 20 & 16,000 \\
\hline & & 17 & $6(35.3)$ & $4(23.8)$ & $4(23.8)$ & $3(23.8)$ & 14 & 52,000 \\
\hline & & 21 & $3(14.3)$ & $6(28.65)$ & $7(33.3)$ & $5(23.8)$ & 16 & 31,000 \\
\hline 5 & $\begin{array}{l}62 \text { years, female, bone } \\
\text { defect }\end{array}$ & 18 & $4(22.2)$ & $5(23.8)$ & $5(23.8)$ & $4(23.8)$ & 17 & 43,000 \\
\hline$M \pm S D$ & & $15.8 \pm 4.5$ & $\begin{array}{c}3.8 \pm 1.3 \\
(25.3 \pm 8.8)\end{array}$ & $\begin{array}{c}5.2 \pm 1.3 \\
(34.9 \pm 11.6) \\
\end{array}$ & $\begin{array}{c}3.8 \pm 2.4 \\
(22.0 \pm 9.2)\end{array}$ & $\begin{array}{c}2.8 \pm 1.8 \\
(16.4 \pm 7.1) \\
\end{array}$ & $16.2 \pm 2.5$ & $34,200.0 \pm 13,809.4$ \\
\hline
\end{tabular}

Note: $\mathrm{SCl}$ - spinal cord injury.

the use of a thin layer of collagen hydrogel as a substrate. Outgrowth of human NC-MSCs was observed from more than half of the explanted HFs. Herewith, most of the HFs revealed the mixed outgrowth of NCMSCs and keratinocytes (34.9 $\pm 11.6 \% \mathrm{HFs}$ vs $25.3 \pm$ $8.8 \% \mathrm{HFs}$ with pure outgrowth of NC-MSCs). In our case, the emigration and proliferation of NC-MSCs from explants have started 5-10 hours, the culture time of the primary culture was $16.2 \pm 2.5$ days on the average and $10^{4} \mathrm{NC}-\mathrm{MSC}$ were obtained from a single $\mathrm{HF}$, which corresponds to the data published by other groups $[15,16]$. Fig. 1, a shows the HF explant with a pure outgrowth of NC-MSCs just before subculturing. Fig. 1, $b$ shows the NC-MSCs with characteristic stellate morphology and migratory behavior. For further expansion of pure cultures, the NC-MSCs were successfully separated from the primary keratinocyte impurity by a differential trypsinization technique while subculturing. The use of 0.01 trypsin solution allowed the NC-MSCs to be effectively collected, while dense colonies of keratinocytes remained attached to the substrate. The absence of NC-MSCs contamination by keratinocytes at $\mathrm{P} 1$ was verified by immunostaining for pan-cytokeratin, which gave a negative result (data not shown).

Beginning the $\mathrm{P} 1$ in our culture conditions, a stable rapid growth of NC-MSCs was established. Cell growth parameters at P1-P3 are shown in Table 2. Thus, when the seeding concentration was in the range of $500-1300$ cells per $1 \mathrm{~cm}^{2}$, the cultures reached
70-100\% confluency within 5-7 days, and been subcultured every week. At the same time, an average $6.04 \pm 0.35$ PDN took place within a passage, with averaged PDT $27.90 \pm 1.58$ hours. These parameters during the early passages (P1-P3) showed weak differences both within the culture from one donor at different passages, and between donors.

Determination parameters of safety and efficacy of human cell-based medicinal product mean the stability of the cell karyotype after in vitro expansion and the number of CFU. Cultures from all donors at the end of expansion were subjected to cytogenetic analysis and showed normal karyotype. Analysis of the CFU frequency showed that after large-scale expansion of cultures of human adult NC-MSCs from HF, a significant number of cells maintained the clonogenic potential and CFU frequency was $25.9 \pm 7.7 \%$. An important feature of stem cells is the ability to self-renew. To assess the self-renewal, five CFUs per each donor were subcloned. In all cases the formation of secondary colonies was detected. Thus, human adult NC-MSCs from $\mathrm{HF}$ retain their clonogenic potential and ability to self-renew after large-scale expansion under our cell culture cultural conditions.

We also examined the phenotype of the obtained cultures by flow cytometry and immunocytochemistry for key markers of neural crest (Sox10, p75), common markers of stem cells (nestin, Sox2 and CD34), markers of mesenchymal stem/stromal cells (CD73, CD90 and CD105), receptors for some growth factors

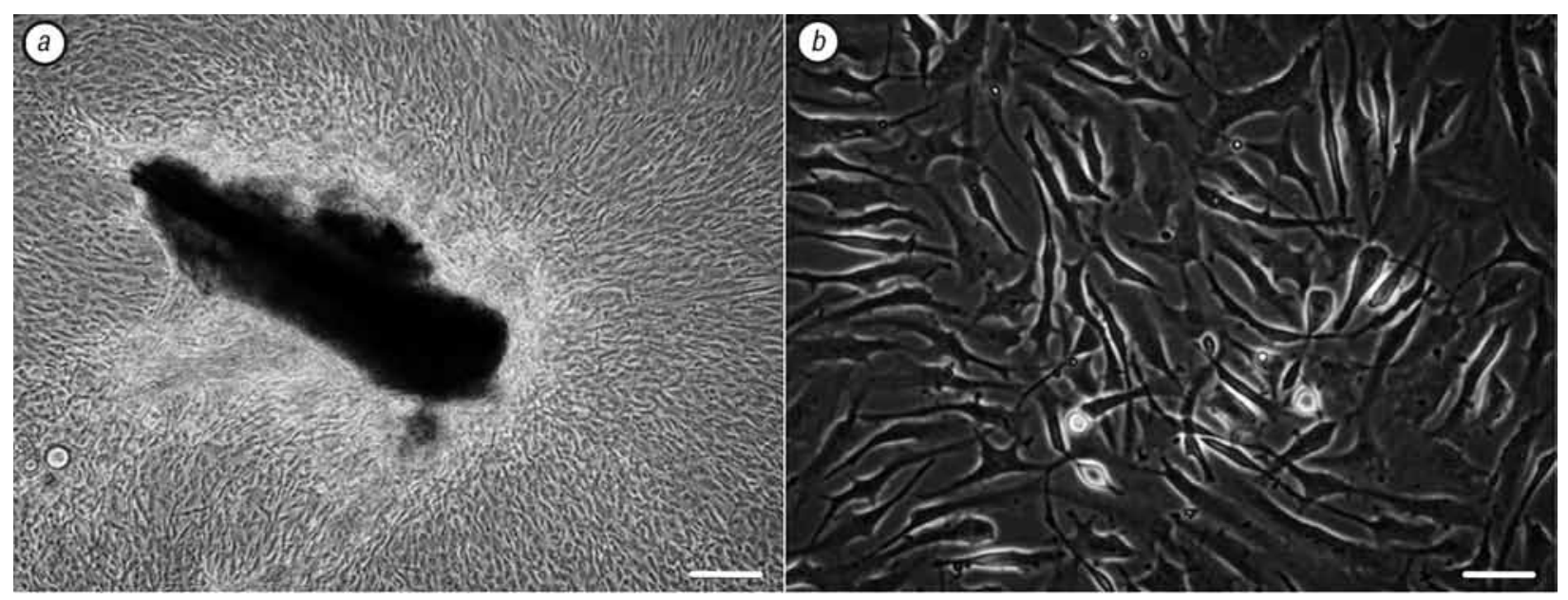

Fig. 1. Adult NC-MSCs from HF: a - explant of HF with pure outgrowth of NC-MSCs just before passaging. Phase contrast, scale bar $-200 \mu \mathrm{m} ; b-$ characteristic stellate morphology of NC-MSCs. The small rounded bright cells - NC-MSCs in mitosis. Phase contrast, scale bar $-50 \mu \mathrm{m}$ 
Table 2. Parameters of cell cultures at P1-P3

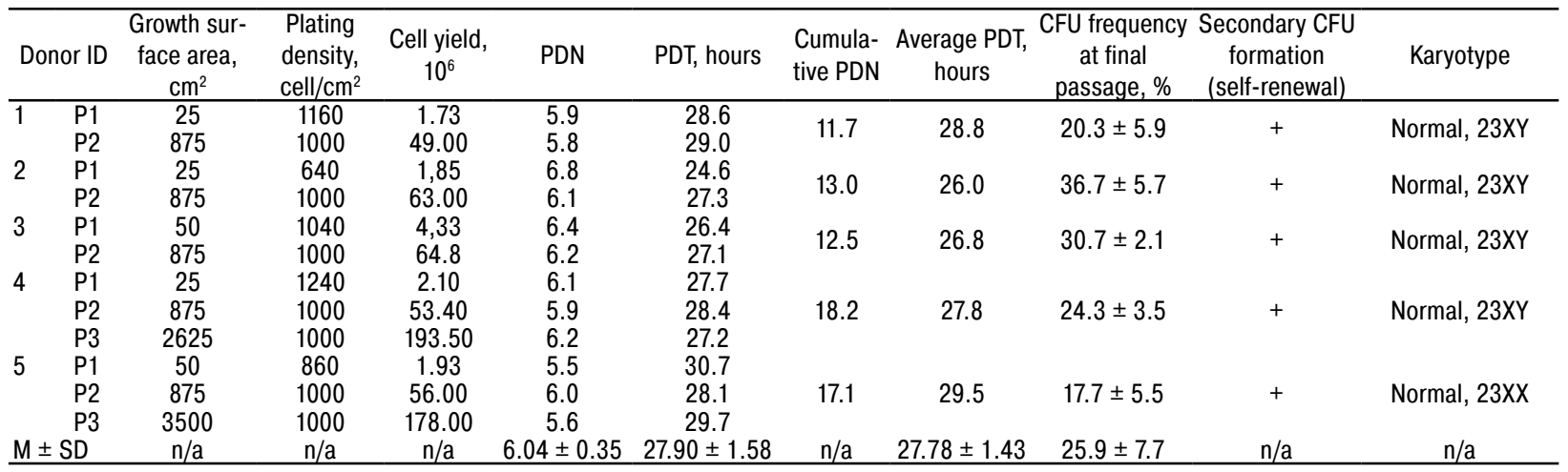

Note: $\mathrm{n} / \mathrm{a}-$ not applicable.

(CD140a/PDGFRa, CD140b/PDGFR $\beta$ and CD349/ Frizzled-9), adhesion molecules (CD56/NCAM, CD146/MCAM and CD166/ALCAM), and markers of hematopoietic (CD45) and antigen-presenting cells (HLA-DR). Representative flow cytometry histograms of cell culture from donor \# 2 are shown in Fig. 2. Summary data for all donors are presented in Table 3.

All expanded cultures of NC-MSCs contain more than $90 \%$ Sox $10^{+}$and Nestin ${ }^{+}$cells, but the number of cells expressing p75 (CD271) varied significantly from 45.9 to $93.8 \%$ positive cells $(73.32 \pm 18.39 \%$ ). Interesting, NC-MSCs homogeneously expressed the key mesenchymal stem/stromal markers CD73, CD90 and CD105 $[2,3]$. All these markers were presented on more than $90 \%$ cells in average. Also in average more than $90 \%$ of the cells were positive for CD140b, CD166 and CD349. More heterogeneous cultures were by expression of CD140a (67.68 \pm 14.26$)$ and CD146 (87.92 \pm 8.42 ). Adult NC-MSCs were negative for hematopoietic markers CD34 and CD45 (the number of positive cells did not exceed $1 \%$ ). Sometimes a small number of CD56 (up to $7.8 \%$ ) and HLA$\mathrm{DR}^{+}$(up to $4.2 \%$ ) cells was noted.

Taking into account the key role of Sox10, p75 (CD271) and nestin in the determination of the identity of NC-MSCs [4, 5], we also examined them using the immunocytochemical analysis. Additionally, Sox2 has also been studied because of its important role in stemness maintenance and functional properties of NC-MSCs $[20,21]$. As shown in Fig. 3 and
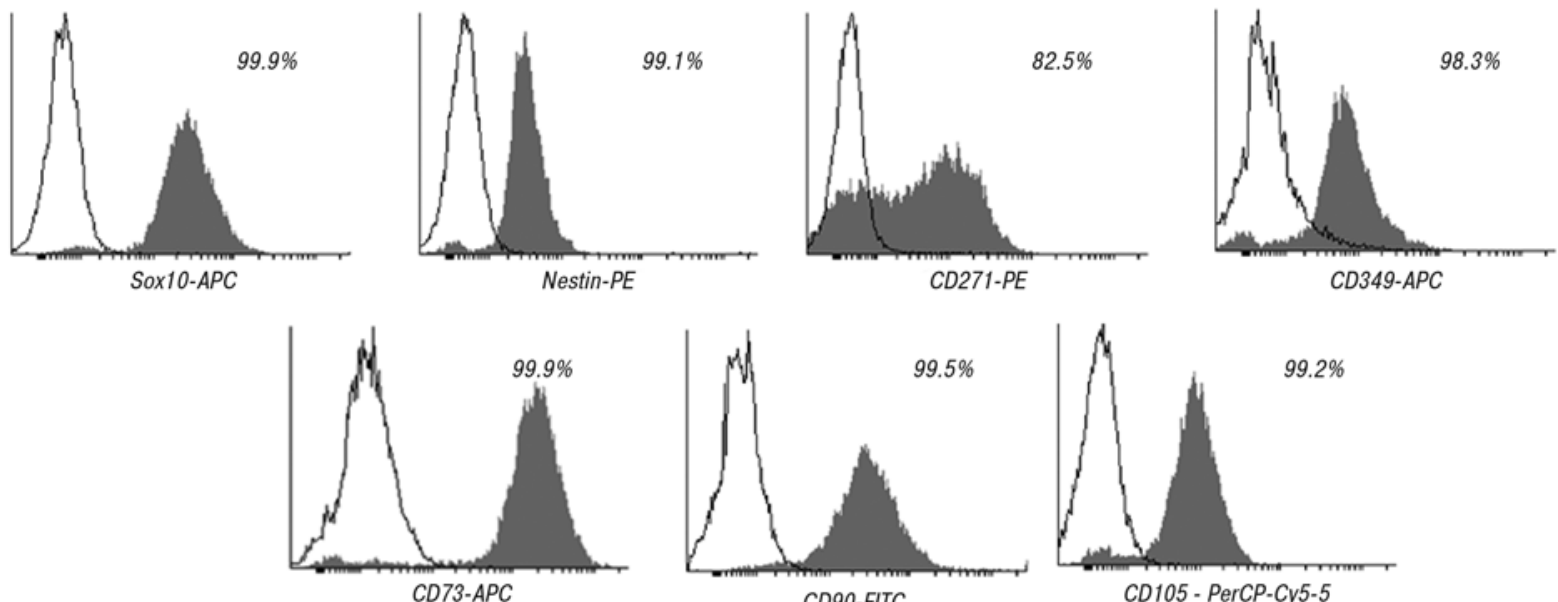

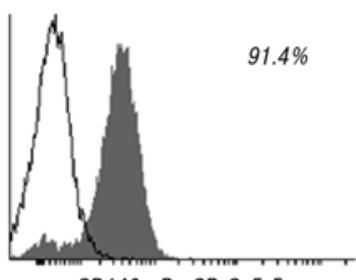

CD140a-PerCP-Cy5-5

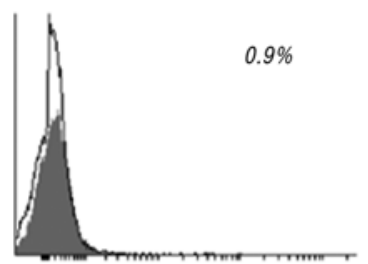

$C D 34-A P C$

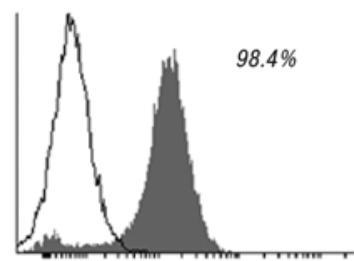

CD140b-PE

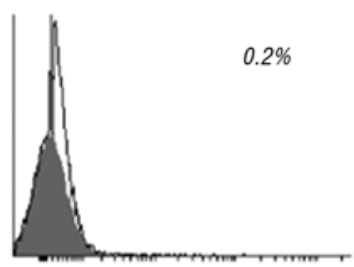

CD45-FITC

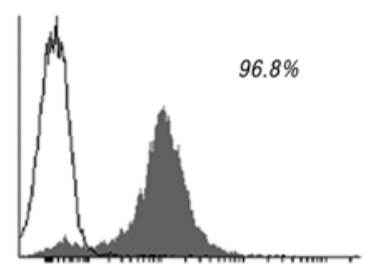

CD146-PE-CF594

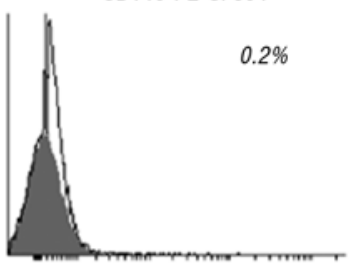

CD45-FITC

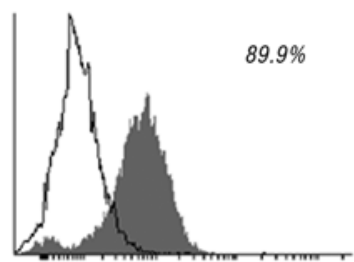

CD166-BV421

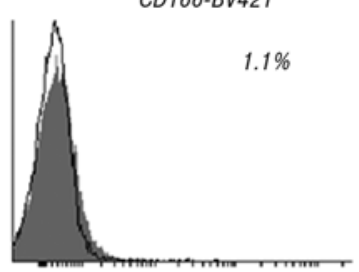

HLA-DR-PE-Cy 5

Fig. 2. Representative results of NC-MSCs phenotype analysis by flow cytometry 
Table 3. Phenotype of NC-MSCs at last passage (P2-P3) by flow cytometry

\begin{tabular}{|c|c|c|c|c|c|c|c|c|c|c|c|c|c|c|c|}
\hline Donor ID & Sox10 & $\begin{array}{l}\text { 271 (p75) } \\
\end{array}$ & Nestin & CD73 & CD90 & CD105 & CD140a & CD140b & CD146 & CD166 & CD349 & CD34 & CD45 & CD56 & HLA-DR \\
\hline 1 & 95.3 & 65.3 & 97.8 & 99.5 & 98.7 & 96.3 & 62.8 & 96.9 & 82.3 & 91.5 & & 0,3 & 0,6 & 1,5 & 1,2 \\
\hline 2 & & & & & & & & & & & & & & & \\
\hline 3 & 96 & & & & & & & & & & & & & & \\
\hline 4 & 98 & & 97 & 99 & 96. & 98 & & 94. & & 98 & & 1 , & & & 0 \\
\hline & 91.8 & & 88.7 & 96.9 & 97.3 & 96.4 & 69.7 & 81.7 & 86.3 & 97. & & 0,4 & 0,1 & 2,4 & 4,2 \\
\hline & $96.30 \pm$ & $73.32 \pm$ & $95.04 \pm$ & $98.16 \pm$ & $97.00 \pm$ & $96.06 \pm$ & $67.68 \pm$ & $91.84 \pm$ & $87.92 \pm$ & 93.0 & $93.06 \pm$ & $0.74 \pm$ & $0.32 \pm$ & $3.08 \pm$ & $1.56 \pm$ \\
\hline & 3.09 & 18.39 & 4.44 & 1.98 & 2.63 & 3.09 & 14.26 & 7.10 & 8.42 & 4.84 & 4.37 & 0.38 & 0.22 & 2.90 & 1.51 \\
\hline
\end{tabular}

Fig. 4, most of the cells in the culture were Sox $10^{+} \mathrm{p} 75^{+}$ and Sox $2^{+} \mathrm{Nestin}^{+}$, which confirms the flow cytometry data. As described earlier, for human adult NC-MSCs from HF $[16,22]$ the Sox10 showed a characteristic nuclear-cytoplasmic localization, consistent with its role as a transcription factor and active nucleocytoplasmic shuttle protein [23]. Sox2 had predominantly nuclear localization, which is characteristic of its function as a transcription factor. Nestin and p75 also showed the characteristic staining for the intermediate filament protein and intracellular domain of growth factor receptor respectively.

In addition to the ability to self-renew another important functional property of stem cells is the ability to multilineage differentiation. Considering that HF from the head area were used in our study, these NC-MSCs were derived from the cranial neural crest during the embryonic development. Cranial neural crest gives rise to the widest variety of tissues and cell types from all the axial domain of neural crest [5]. So, in addition to melanocytes, neurons and glia of the peripheral nervous system, the cranial neural crest gives rise to ectomesenchyme, from which a part of the bone, cartilage and connective tissue of the head and neck is formed. Based on this, we verified the identity of the obtained adult NC-MSCs by ability to differentiate into both mesenchymal cell types (adipocytes and osteoblasts), and into neurons and Schwann cells. Adult NC-MSCs were cultured for 14 days in an adipogenic induction medium and have successfully differentiated into the adipocytes containing lipid vacu-

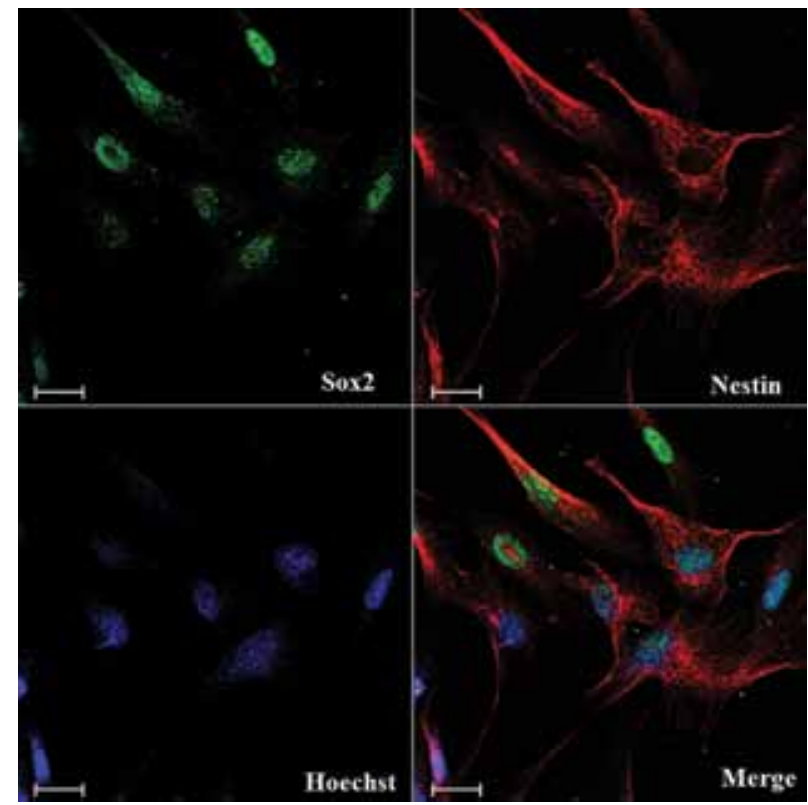

Fig. 3. Immunocytochemical analysis of NC-MSCs for Sox2 (green) and Nestin (red) expression. Scale bar $-20 \mu \mathrm{m}$ oles (Fig. 5, a). When cultured in osteogenic medium during 14 days, NC-MSCs were converted to alkaline phosphatase-positive osteoblasts (Fig. 5, b) and after 21 days the mineralized extracellular matrix was produced (Fig. 5, c). When NC-MSCs were cultured in the presence of a synthetic analogue of retinoic acid and neurotrophins (NGF, BDNF and GDNF), they were converted into the homogeneous population of neuronal $\beta$-III-tubulin positive cells with spherical or oval soma and long thin processes (Fig. 5, d). Under the influence of neuregulin-1, PDGF-BB and the activation of cAMP signaling by forskolin, NC-MSCs differentiated into $S 100 \beta$-positive cells with the characteristics for Schwann cells bipolar morphology (Fig. 5, e).

As mentioned in the Introduction, the existence of adult NC-MSCs in the bulge region of HF was first shown by M. Siber-Blum using whisker follicles from Wnt1-Cre transgenic mice for tracing developmental origin of this cell population from neural crest [11]. In the study NC-MSCs obtaining was based on the migratory behavior of this cell type. NC-MSCs emigrated and began to proliferate rapidly while culturing the explants of the bulge region on collagen substrate in a special neural crest cell growth medium. Mouse NC-MSCs from bulge region of HF were Sox $10^{+} \mathrm{Nestin}^{+}$and had the ability for directed differentiation into neurons, Schwann cells, melanocytes, smooth muscle cells and chondrocytes. In parallel, Fernandes et al. using the same Wnt1-Cre transgenic mouse line reported the isolation of another cell population of the neural crest origin from derma of facial skin, which was called skin-

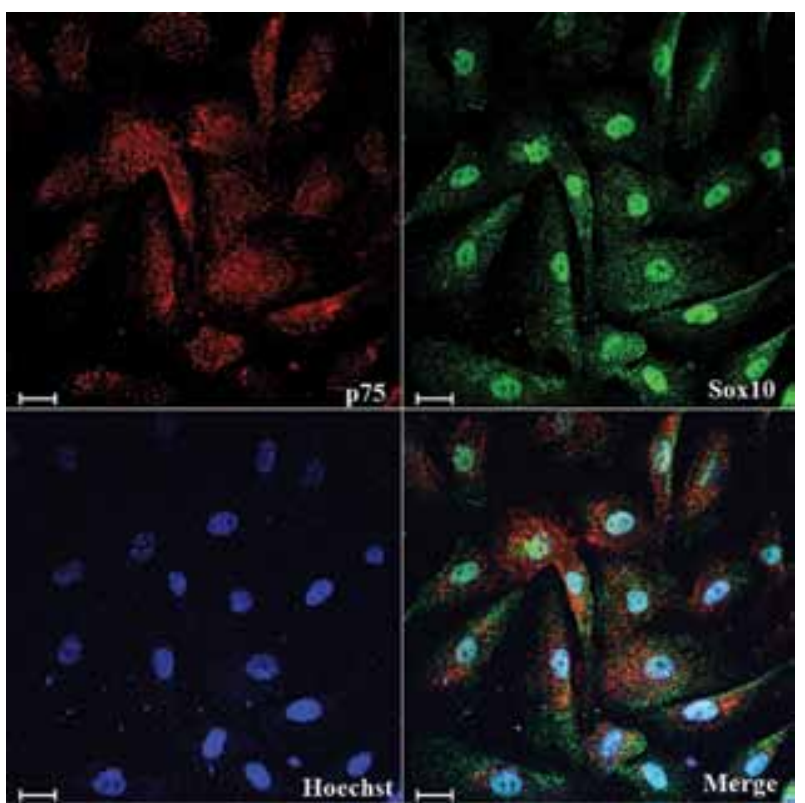

Fig. 4. Immunocytochemical analysis of NC-MSCs for p75 (CD271) (red) and Sox10 (green) expression. Scale bar $-20 \mu \mathrm{m}$ 

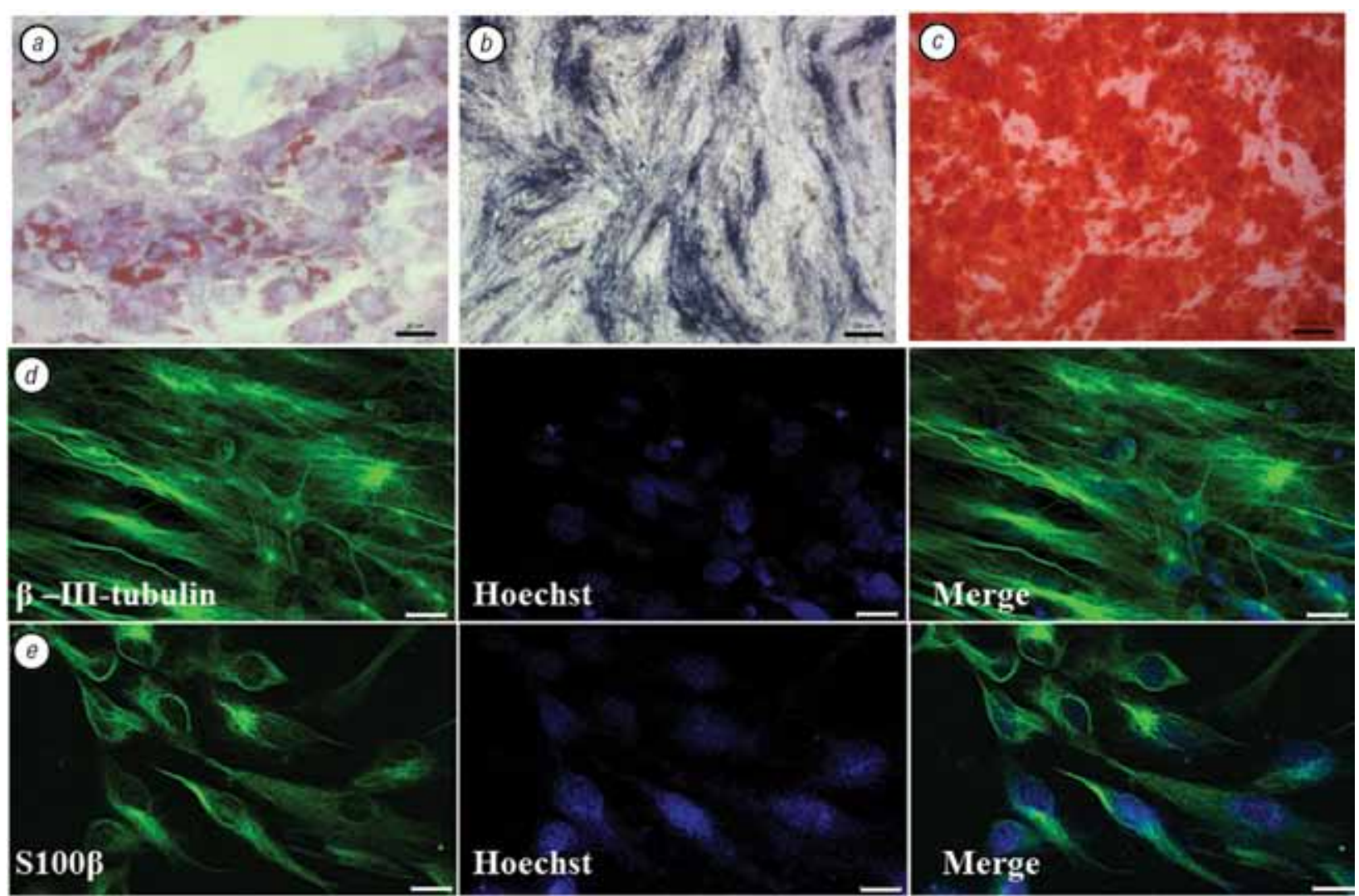

Fig. 5. Detection of NC-MSCs directed multilineage differentiation: a - adipogenic differentiation, Oil Red O staining of lipid vacuoles (red). Scale bar - $50 \mu \mathrm{m} ; b$ - osteogenic differentiation, BCIP/NBT staining for ALP activity (dark blue). Scale bar - $100 \mu \mathrm{m}$; $c$ - osteogenic differentiation, Alizarin Red S staining of mineralized extracellular matrix (red). Scale bar $-200 \mu \mathrm{m} ; d-$ neuronal differentiation, immunocytochemical detection of $\beta$-III-tubulin (green). Scale bar $-20 \mu \mathrm{m} ; e-$ glial differentiation (Schwann cells), immunocytochemical detection of $S 100 \beta$ protein (green). Scale bar $-20 \mu \mathrm{m}$

derived precursors cells (SKPs) [24]. It turned out that the tissue niche for SKPs is dermal papilla of HFs from facial skin and whisker follicles [24]. The procedure for SKPs obtaining was based on their ability to grow in the form of spheres (resembling neurospheres) under serum-free and non-adherent conditions. At the same time, SKPs showed a similar differentiation potential to NC-MSCs from the bulge region of HF. However, they were Sox 10 and p 75 negative, but they expressed other characteristic neural crest markers - Nestin, Slug, Snail, Twist and Sox9 [24]. Later, a more in-depth analysis of the localization of NC-MSCs in whisker follicles and HF from dorsal and ventral skin of mice has been made with use of Wnt1-Cre, Ht-PA-Cre, Sox 10'acz, Dhh-Cre and Dct-Cre transgenic mouse lines [12]. In those paper it was confirmed that many structures of whisker follicle derived from neural crest, harbor cells with stem cells-like properties and this cells linked to mesenchymal, glial and melanocyte lineages. Such structures in whisker follicles are: bulge region, dermal sheath, capsule, dermal papilla, nerve terminals and regions above sebaceous glands. Analysis of mouse $\mathrm{HF}$ from back skin showed that neural crest-derived cells are localized in the bulge region and in the bulb above the dermal papilla [12]. The dermal papilla of the back skin itself does not originate from the neural crest. Lineage tracing analysis showed that neural crest-derived cells in trunk HFs are linked to glial and melanocyte lineages. Using somewhat different from
Fernandes et al. [24] growth conditions, the authors obtained spheres from mouse back skin and human skin (face and thigh) samples [12]. Analysis of the cell composition of the spheres obtained from transgenic mice showed that they are formed by cells that have neural crest origin. It should be noted, $100 \%$ mouse and human skin-derived spheres contains $\mathrm{p} 75^{+}$and Sox $10^{+}$cells. In mouse spheres $67.0 \pm 10.5 \%$ of all cells expressed $p 75 ; 76.6 \pm 4.5 \%$ of all cells expressed Sox 10 ; and $58.6 \pm 10.5 \%$ of all cells were double positive for $p 75$ and Sox 10 . Then, $15.0 \pm 6.2 \%$ of all sphere cells were negative for these markers, which indicates cell heterogeneity in the composition of spheres, which may be associated with spontaneous cell differentiation. Similar to mouse spheres, all human spheres contained p75/Sox 10 double positive cells, which accounted for $>60 \%$ of all cells. However, the cells were negative for other neural crest markers, such as Sox9 and HNK-1 (CD57). Both mouse and human neural crest-derived cells from HF showed the ability to multilineage directed differentiation into the main derivatives of the neural crest: peripheral neurons, Schwann cells, melanocytes, adipocytes, chondrocytes and smooth muscle cells [12].

Thus, lineage tracing experiments using transgenic mouse lines showed that the HF contains various Sox 10 and p75 positive and negative cells of neural crest origin with multilineage differentiation potential and stem cell-like properties. 
The existence of NC-MSCs in the bulge region of human HF was also confirmed by other authors and several variants of growth media and methods for their isolation were proposed for the isolation of NC-MSCs from dissociated HFs or skin samples and their growth under the floating sphere conditions [14, 17, 18].

Although it has been shown in [12] that the human skin-derived spheres could be serially subcultured for more than 3 months and generates more than $10^{9}$ cells during this time, the disadvantage of human NC-MSCs culturing under sphere conditions is its high cost and relatively low cell growth rate.

For these reasons, attempts have been made to modify the original M. Siber-Blum protocol to obtain NC-MSCs cultures from human HF explants. In one of the first reports it was shown that NC-MSCs do not provide cell emigration and growth in a monolayer when human HF explants culturing, but only form spheroid structures attached to the bulge area of HFs $[14,25]$. Later, the group of M. Siber-Blum and other authors reported the successful adaptation of the explant method to the production of human NC-MSCs cultures $[15,16,22]$. At the same time, several critical moments and shortcomings in the use of this technique were revealed. The most critical and time-consuming step in adult NC-MSCs obtaining is the establishment of primary cultures $[15,16,22]$. The main difficulties in obtaining primary cultures of human adult NC-MSCs from HF explants are the detachment of HFs, the absence of outgrowth of NC-MSCs and overgrowth of keratinocytes $[15,16]$. Also there are contradictory data on NC-MSCs morphology (from spindle-like to stellate) and markers expression $[15,16]$. It can be due to both the use of different culturing conditions and the methods of extraction of HFs from a skin (dissected vs plucked) and different skin regions. It should be noted that the equivalence of the use of dissected vs plucked human HFs for NC-MSCs isolation and the identity of the resulting cell populations remain an open question. There is an opinion that the bulge region is damaged in plucked HFs. As a result, various phenotypes of adult NC-MSCs derived from plucked and dissected HF have been reported. So, in [15], the NC-MSCs from plucked HFs were Sox10 negative, but positive for common used neural crest markers, like Nestin, Slug, AP-2 $\alpha$ and Sox9. Whereas $\mathrm{NC}$-MSCs the resulting from the dissected HFs were So $10^{+}$Sox $2^{+} \mathrm{Nestin}^{+}$by immunocytochemistry [16, 22]. There was also expression of Slug, Twist and $P 75$ genes detected by qPCR $[16,22]$.

In our study, to improve the reliability and effectiveness of the procedure for NC-MSCs obtaining, we modified the original protocol of M. Siber-Blum [11] in the following points: 1) use of Dispase enzyme for separation of the epidermis and facilitation of the HFs dissection procedure; 2) use of a thin layer of collagen hydrogel as an adhesive coating for the attachment of HF explants; 3) maintenance of adhered explants in the primary culture until the moment of subculturing. In fine, these minor modifications have significantly improved cell isolation and culturing protocol, which will be discussed below.

So, we managed to significantly reduce the frequency of HF explants detaching to less than $20 \%$. For comparison, when using the poly-D-lysine treated plastic surface, more than $40 \%$ of explants of HFs [15] are detached.

Regardless of the cell culture conditions used, it has not yet been possible to obtain $100 \%$ outgrowth of NC-MSCs from all adhered HFs and the reasons for this remain unclear. A possible link can exist with the growth phase of HF. So in [16], it is shown that more often NC-MSCs emigrated from explanted HFs been in early or late anagen growth stages. On the other hand, in [17] it has shown the possibility of NC-MSCs obtaining both from HFs in the anagen stage and the telogen stage. In various studies, the outgrowth of NC-MSCs was from $20 \%$ in [25] to $34 \%$ in [15] or almost for $50 \%$ of explants [16]. Clewes et al. [16] were noted that more often emigration of human NC-MSCs was observed from the explants of HFs with preserved dermal sheath $-45.3 \pm 13.0 \%$. NC-MSCs did not emigrate from the separately cultured dermal sheath of HFs and emigrated only from $6.3 \pm 6.3 \% \mathrm{HFs}$ without dermal sheath. Thus, the role of the dermal sheath is probably to preserve the bulge region unaffected (intact, uninjured) during the extraction and isolation of HFs.

Keratinocytes outgrowth from human HF explants is a common problem $[15,16]$. Interestingly, the emigration of keratinocytes from the mouse bulge region of whisker follicles is observed much later than the emigration of NC-MSCs [11], or can be excluded altogether by using a selective growth medium [26]. At the same time, keratinocytes outgrowth is often observed when culturing of rat bulge whisker follicle explants (our unpublished observations) or human HF explants. So, in [15] reported the keratinocytes outgrowth was from $33-100 \%$ explanted HFs, which is comparable to our data. Further progress in this direction may be related to the development of a selective growth medium, although according to our observations, keratinocytes proliferate in the primary culture of explanted HFs even in the absence of EGF in the growth medium. It should be noted that the problem of contamination of NC-MSCs cultures by keratinocytes with further expansion can be successfully solved using the technique of differential trypsinization, as it was made in our presented work.

As for the phenotype of adult NC-MSCs obtained during the large-scale expansion, it is comparable to that described for cultures obtained from dissected human HFs $[16,17,22]$ and differs from that described for cultures obtained from plucked human HFs [15]. The reason for this can be both the HF isolation methods and slightly different culture conditions. A weak modification of growth conditions can either influence the expression of neural crest markers (like Sox9, Sox10 or p75), or give selective benefits to different cell populations with neural crest origin, 
which contains in HF. So, Sox 10 and p75 are the first markers that distinguish neural crest cells immediately after their induction from Sox $2^{+}$neuroepithelium of neural tube [27]. And although these markers are used for prospective isolation and/or for the confirmation of neural crest origin of cells from different tissues of the adult organism, their expression is not always observed in adult neural crest-derived cells, especially in ectomesenchymal cells of the craniofacial region $[4,5]$. Thus, expression of Sox 10 by neural crest-derived cells at later stages of development and in adulthood can be related to their broader differentiation potential while retaining the ability to acquire neuronal, glial and melanocyte fates [28, 29]. Whereas ectomesenchymal cells with differentiation potential restricted to mesenchymal cell fate can be Sox 10 negative $[4,5]$. The p75 function and the heterogeneity of its expression in the populations of adult neural crest-derived cells remain largely not understood. A comparative study of the proliferation and differentiation potential of Sox $10^{+} \mathrm{p} 75^{+}$and Sox $10^{+} p 75^{-}$cells is needed. Concerning the double negative Sox $10^{-} \mathrm{p} 75^{-}$cells, we assume that they can be progenitors with the development of potential restricted to mesenchymal cell fate [30]. This is supported by the preservation of the expression of CD73, CD90 and CD105 (markers of mesenchymal stem/ stromal cells) by Sox $10^{-} \mathrm{p} 75^{-}$cells.

An important point is that the NC-MSCs expanded by our protocol are Sox $2^{+}$. Sox 2 is one of the main factors of pluripotency and a marker of neural stem/progenitor cells of central nervous system. Although at the time of induction, neural crest cells are Sox 2 negative $[27,30]$, its expression appears in them at later stages of development and is associated with the preservation of multipotency and ability to neurogenesis $[4,5$, $20,30]$. Detection of a small number of $\mathrm{CD}^{2} 6^{+}$cells may indicate spontaneous neuronal differentiation of NC-MSCs during expansion. It is important to note that in addition to neurogenesis, Sox $2^{+} \mathrm{NC}-\mathrm{MSCs}$ play a key role in skin wound healing and in reparative regeneration process regulation as shown in the experiments with use of transgenic mouse lineages [21].

Also important findings in our study are the expression on the adult NC-MSCs of such receptors for growth factors as CD140a (PDGFRa), CD140b (PDGFRß), CD349 (Frizzled-9) and CD166 (ALCAM). Expression of CD140a and CD140b by NC-MSCs can be associated with several key aspects of their biology, such as differentiation into Schwann cells, or their activation after tissue damage through platelet-released PDGFs. The role of PDGF proteins in NC-MSCs biology should also be studied in more details in the light of the possible use for cell xeno-free expansion of a platelet lysate which contains them in large quantities. Concerning CD349 expression, this indicates the need for Wnt-signaling studying in adult NC-MSCs biology. It is known that in the early stages of development the self-renewal of NC-MSCs is supported by the combined action of Wnt and BMP signaling [31]. The role of these factors in the proliferation, self-renewal and differentiation of adult NC-MSCs is poorly understood. CD166 expression may indicate the presence of an immunomodulatory properties in adult NC-MSCs. Detection of the minor population of $\mathrm{HLA}^{-\mathrm{DR}^{+}}$cells can also be associated with the immunological functions of NC-MSCs. It is well known that bone marrow-derived mesenchymal stem/stromal cells have the ability for inducible expression of HLA-DR after exposition to inflammatory signals. This aspect of adult NC-MSCs biology is not actually studied.

Thereof, our study shows for the first time the principal possibility of large-scale expansion of adult NC-MSCs from HF with preservation of their phenotype and basic functional properties. The therapeutic number of cells required for clinical use ranges from $40 \cdot 10^{6}$ to $200 \cdot 10^{6}$ and can be obtained during one month of culturing. These terms theoretically allow us to consider the possibility of developing autologous medicinal products based on human NC-MSCs for the treatment of spinal cord injuries and traumatic peripheral nerve defects not only in the chronic but also in the subacute period. It is generally accepted that reconstructive surgery of damaged spinal cord and peripheral nerve cannot be carried out in the acute period, and the most favorable results are obtained when they are carried out in the subacute/early chronic period in comparison to late chronic period. Previously, despite the positive results of adult NC-MSCs application obtained in animal models of spinal cord injury and peripheral nerve defect, some skepticism is caused by the absence of protocols of obtaining a therapeutic dose in a short period of time (no more than 4-6 weeks). Next steps in this field should be further improvement of the isolation technique from HF, development of xeno-free conditions for adult NC-MSCs expansion and conducting of randomized clinical trials to assess the effectiveness of this cell type in the treatment of various diseases and defects of the neural crest-derived tissues and structures in comparison with existing "gold standards" of surgical or therapeutic treatment for these conditions.

\section{REFERENCES}

1. Regulatory aspects of gene therapy and cell therapy products. A global perspective. MC Galli, M Serabian, eds. Springer, 2015. $230 \mathrm{p}$.

2. Dominici M, Le Blanc K, Mueller I, et al. Minimal criteria for defining multipotent mesenchymal stromal cells. The International Society for Cellular Therapy position statement. Cytotherapy 2006; 8: 315-7.

3. Bourin P, Bunnell BA, Casteilla L, et al. Stromal cells from the adipose tissue-derived stromal vascular fraction and culture expanded adipose tissue-derived stromal/stem cells: a joint statement of the International Federation for Adipose Therapeutics and Science (IFATS) and the International Society for Cellular Therapy (ISCT). Cytotherapy 2013; 15: 641-8.

4. Achilleos A, Trainor PA. Neural crest stem cells: discovery, properties and potential for therapy. Cell Res 2012; 22: $288-304$. 
5. Dupin E, Sommer L. Neural crest progenitors and stem cells: from early development to adulthood. Dev Biol 2012; 366: 83-95.

6. Chung IH, Yamaza T, Zhao H, et al. Stem cell property of postmigratory cranial neural crest cells and their utility in alveolar bone regeneration and tooth development. Stem Cells 2009; 27: 866-77.

7. Amoh Y, Li L, Campillo R, et al. Implanted hair follicle stem cells form Schwann cells that support repair of severed peripheral nerves. Proc Natl Acad Sci U S A 2005; 102: 17734-8.

8. Vasyliev RG, Rodnichenko AE, Shamalo SN, et al. Effects of neural crest-derived multipotent stem cells on regeneration of an injured peripheral nerve in mice. Neurophysiology $2015 ; 47$ : 80-3.

9. Konig N, Trolle C, Kapuralin K, et al. Murine neural crest stem cells and embryonic stem cell-derived neuron precursors survive and differentiate after transplantation in a model of dorsal root avulsion. J Tissue Eng Regen Med 2017; 11: 129-37.

10. Hu YF, Gourab K, Wells C, et al. Epidermal neural crest stem cell (EPI-NCSC) - mediated recovery of sensory function in a mouse model of spinal cord injury. Stem Cell Rev 2010; 6: 186-98.

11. Sieber-Blum M, Grim M, Hu YF, et al. Pluripotent neural crest stem cells in the adult hair follicle. Dev Dyn 2004; 231: $258-69$.

12. Wong CE, Paratore C, Dours-Zimmermann MT, et al. Neural crest-derived cells with stem cell features can be traced back to multiple lineages in the adult skin. J Cell Biol 2006; 175: $1005-15$.

13. Gericota B1, Anderson JS, Mitchell G, et al. Canine epidermal neural crest stem cells: characterization and potential as therapy candidate for a large animal model of spinal cord injury. Stem Cells Transl Med 2014; 3: 334-45.

14. Yu H, Fang D, Kumar SM, et al. Isolation of a novel population of multipotent adult stem cells from human hair follicles. Am J Pathol 2006; 168: 1879-88.

15. Gho CG, Schomann T, de Groot SC, et al. Isolation, expansion and neural differentiation of stem cells from human plucked hair: a further step towards autologous nerve recovery. Cytotechnology 2016; 68: 1849-58.

16. Clewes $O$, Narytnyk A, Gillinder KR, et al. Human epidermal neural crest stem cells (hEPI-NCSC)-characterization and directed differentiation into osteocytes and melanocytes. Stem Cell Rev 2011; 7: 799-814.
17. Yang $\mathrm{R}, \mathrm{Xu} \mathrm{X}$. Isolation and culture of neural crest stem cells from human hair follicles. J Vis Exp 2013; (74). doi: 10.3791/3194.

18. Zong Z, Li N, Ran X, et al. Isolation and characterization of two kinds of stem cells from the same human skin back sample with therapeutic potential in spinal cord injury. PLoS One 2012; 7: e50222.

19. Freshney RI. Culture of animal cells: a manual of basic technique and specialized applications. $6^{\text {th }}$ ed. WileyBlackwell, 2010. 796 p.

20. Cimadamore F, Fishwick K, Giusto E, et al. Human ESC-derived neural crest model reveals a key role for SOX2 in sensory neurogenesis. Cell Stem Cell 2011; 8: 538-51.

21. Johnston AP, Naska S, Jones K, et al. Sox2-mediated regulation of adult neural crest precursors and skin repair. Stem Cell Rep 2013; 1: 38-45.

22. Sakaue M, Sieber-Blum M. Human epidermal neural crest stem cells as a source of Schwann cells. Development 2015; 142: 3188-97.

23. Rehberg $S$, Lischka $P$, Glaser $G$, et al. Sox10 is an active nucleocytoplasmic shuttle protein, and shuttling is crucial for Sox10-mediated transactivation. Mol Cell Biol 2002; 22: $5826-34$.

24. Fernandes KJ, McKenzie IA, Mill P, et al. A dermal niche for multipotent adult skin-derived precursor cells. Nat Cell Biol 2004; 6: 1082-93.

25. Yu H, Kumar SM, Kossenkov AV, et al. Stem cells with neural crest characteristics derived from the bulge region of cultured human hair follicles. J Invest Dermatol 2010; 130: $1227-36$.

26. Vasyliev RG, Rodnichenko AE, Zubov DA, et al. Biological properties of neural crest-derived multipotent stem cells from the bulge region of whisker follicle expanded in new culture conditions. Biopolym Cell 2014; 30: 469-76.

27. Stemple DL, Anderson DJ. Isolation of a stem cell for neurons and glia from the mammalian neural crest. Cell 1992; 71 (6): 973-85.

28. Paratore C, Goerich DE, Suter U, et al. Survival and glial fate acquisition of neural crest cells are regulated by an interplay between the transcription factor Sox 10 and extrinsic combinatorial signaling. Development 2001; 128: $3949-61$.

29. Kim J, Lo L, Dormand E, Anderson DJ. SOX10 maintains multipotency and inhibits neuronal differentiation of neural crest stem cells. Neuron 2003; 38: 17-31. 